\title{
Vaginal Estrogen Therapy for the Treatment of Atrophic Vaginitis
}

\author{
Catherine Lynch, M.D.
}

\begin{abstract}
The prevalence of atrophic vaginitis (AV) is particularly high among postmenopausal women who experience decreased ovarian estrogen production. This common menopausal condition, which is often underreported and underdiagnosed, is caused by estrogen-related changes to the vaginal epithelium and can adversely affect quality of life. An accurate diagnosis of AV and its severity is important in choosing the appropriate treatment. Numerous formulations for estrogen therapy are available, and the safety and efficacy of these preparations for the treatment of AV have been evaluated in randomized controlled clinical trials. Although systemic administration of estrogen is effective in treating acute vasomotor symptoms typically associated with perimenopause and early menopause, standard doses may not be sufficient for the treatment of AV-related symptoms that generally arise after long-term estrogen deficiency. Thus, vaginal estrogen preparations (e.g., creams, tablets, rings) are more often recommended for women with moderate to severe AV. With any estrogen therapy, consideration of the potential adverse effects vs. associated benefits is necessary. Appropriate clinical intervention with vaginal estrogen formulations may safely offer postmenopausal women relief from undesirable symptoms and emotional distress associated with AV.
\end{abstract}

\section{Introduction}

A TROPHIC VAGINITIS (AV) is a common condition postmenopausal women experience due to estrogen deficiency that causes involution of the vaginal tissue, leading to itching, burning, dryness, irritation, and dyspareunia. ${ }^{1}$ Unlike vasomotor symptoms that typically accompany menopause, AV symptoms do not diminish over time and are unlikely to resolve without treatment. ${ }^{2}$ Symptoms and consequences of AV can cause years of vulvovaginal discomfort, with a significant impact on quality of life for women in the postmenopausal stage of life.

Estradiol, the main estrogen produced by the ovaries, plays a vital role in keeping the vaginal tissues lubricated and healthy. From puberty, estrogen stimulation maintains a thick vaginal epithelium and production of glycogen. Lactobacilli depend on glycogen from sloughed vaginal cells, and the lactic acid produced by these bacteria maintains vaginal $\mathrm{pH}$ between 3.5 and 4.5 , thus protecting against vaginal and urinary tract infections (UTIs). ${ }^{1,3}$ As estrogen levels drop after menopause, the vaginal tissue becomes atrophic (i.e., thin, dry, and shrunken). Vaginal secretions decline, making sexual activity painful, and women may also experience vaginal discharge. Urinary tract epithelia are also estrogen dependent, reflecting the common embryological origin of the urinary and genital tracts; therefore, urinary tract symptoms, such as dysuria, UTI, and stress incontinence, may also emerge after menopause. ${ }^{1,4}$ These physiological changes increase the likelihood of trauma, infection, and pain for women and can lead to dyspareunia, ultimately resulting in a vaginal surface that is friable, with petechiae, ulcerations, tears, and bleeding occurring from minor contact. 5,6

\section{Epidemiology}

Despite its high incidence, urogenital atrophy is an underreported and underdiagnosed condition. ${ }^{3}$ Few women seek medical attention for vulvovaginal symptoms, often because they are uncomfortable talking about such a private issue. An estimated $10 \%-40 \%$ of postmenopausal women and $10 \%-$ $25 \%$ of women receiving systemic hormone therapy experience urogenital atrophy ${ }^{7}$; however, only $20 \%-25 \%$ seek medical attention. ${ }^{8,9}$ In a self-reported survey of urogenital symptoms among postmenopausal women aged 50-79 years, the prevalence rates of various $\mathrm{AV}$ symptoms have been found to be as follows: vaginal or genital dryness, $27 \%$; vaginal or genital irritation, $18.6 \%$; vaginal or genital discharge, $11.1 \%$; dysuria, $5.2 \% .{ }^{10}$ Symptoms were most common in the Hispanic population, obese women, and those suffering from diabetes mellitus. ${ }^{10}$ Others have reported that $43 \%$ of women

Division of General Obstetrics and Gynecology, Department of Obstetrics and Gynecology, University of South Florida, Tampa, Florida. 
at age 61 years experience vaginal dryness, and 10\% experience burning. ${ }^{11}$ There have also been reports that only $3 \%$ of women of reproductive age are troubled by vaginal dryness, and the incidence increases to $4 \%$ during the early menopausal transition, $21 \%$ in the late menopausal transition, and $47 \%$ during the first 3 years postmenopause. ${ }^{12}$ With a rise in life expectancy, most women could spend about a third of their life in the postmenopausal period. Given the magnitude of this problem, more attention should be focused on the problems faced by women in this age group.

\section{Pathophysiology}

The decline in levels of circulating estrogen associated with the menopausal transition is the etiology in almost all cases of AV. ${ }^{1}$ External causes for the interruption of ovarian estrogen production in premenopausal women include radiation therapy, chemotherapy, and immunological disorders. ${ }^{1}$ Additionally, postpartum decline in estrogen levels, lactation and breastfeeding, surgical removal of the ovaries, and medication used to decrease estrogen levels (in women who have conditions such as uterine fibroids and endometriosis) can also cause AV.

Although some women experience AV shortly after menopause or during short-term estrogen depletion, symptoms of $\mathrm{AV}$ are more likely to arise after a long-term decrease in estrogen stimulation. ${ }^{1}$ The progressive changes in vaginal tissue, including atrophy of the estrogen-responsive epithelium, depletion of vaginal glycogen, alteration in vaginal flora, and the resulting rise in vaginal $\mathrm{pH}$, develop over months or years. ${ }^{1,4,9,13}$ Atrophic changes generally occur later than other menopausal symptoms because relatively lower levels of estrogen are required to maintain healthy urogenital tissues than are required to prevent other estrogen-related symptoms (such as vasomotor symptoms). Because endogenous estrogen levels are markedly lower than those required to stimulate endometrial proliferation at this stage of menopause, a therapeutic window exists during which estrogen can be administered at lower than commonly prescribed doses for the effective treatment of AV (Fig. 1). ${ }^{14}$

The vaginal epithelium is sensitive to estrogen, well rugated, and abundant with glycogen. ${ }^{13}$ The epithelial cells exfoliate constantly, producing lactic acid and other chemicals, such as $\mathrm{H}_{2} \mathrm{O}_{2}$, that control the microflora in the vaginal ecosystem. The interplay of hormones and bacteria in the vagina helps maintain an acidic vaginal $\mathrm{pH}$ of $3.5-4.5$ in healthy mature women, providing natural protection against vaginitis and UTIs. ${ }^{13}$

Vaginal epithelia are estrogen dependent and adversely change in an estrogen-deprived environment. Perimenopausal estradiol levels hover around $120 \mathrm{ng} / \mathrm{L} .{ }^{13}$ After menopause, these levels decrease to approximately $18 \mathrm{ng} / \mathrm{L}$. As estrogen levels decrease at menopause, the vaginal epithelium becomes thin and pale with fewer rugae, with an accompanying progressive loss of vascularity in the vaginal mucosa. Concurrent changes occur as collagen fibers in the vagina swell, fuse, and undergo hyalinization; elastic fibers fragment; and connective tissue increases. The vagina loses elasticity and distensibility, with secondary shortening and narrowing in shape. Symptoms accompanying these changes may include granulation, fissures, ecchymoses, telangiectases, and ulcerations, as well as decreased vaginal secretions and decreased lubrication. These changes may contribute to dyspareunia and sexual dysfunction and can ultimately culminate in vaginal contraction and rigidity. ${ }^{13}$ Another postmenopausal change resulting from the hypoestrogenic

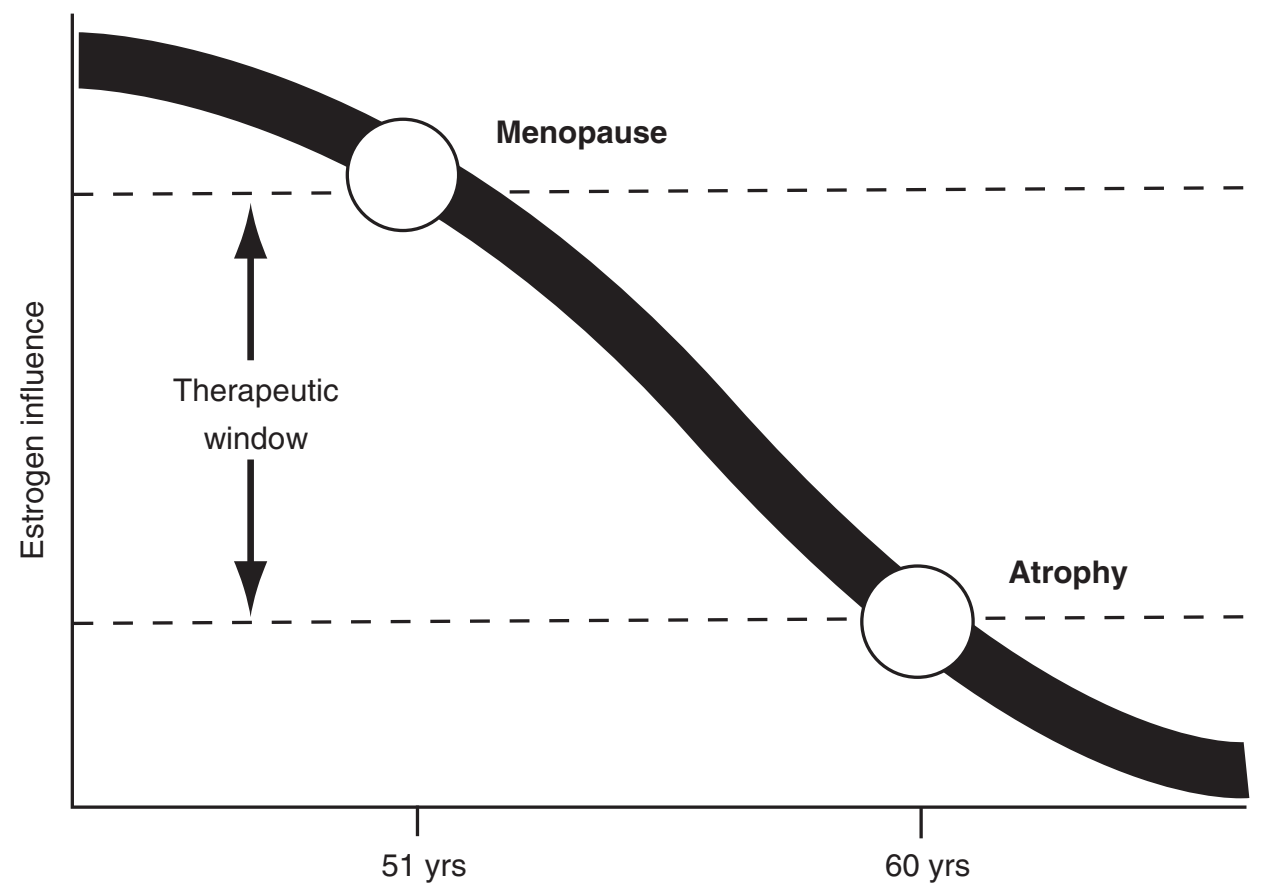

FIG. 1. Long-term, progressive estrogen deficiency leads to atrophy of urogenital tissues. (Adapted from Am J Obstet Gynecol, Samsioe G, Urogenital aging-A hidden Problem, S245-S249, Copyright 1998, with permission from Elsevier). ${ }^{14}$ 
Table 1. Symptoms ANd Signs of Atrophic VAGINITIS $^{1,9,13,16}$
Burning

Tenderness

Dryness

Pressure

Lack of lubrication

Itching

Soreness

Dyspareunia

Vaginal discharge
Genital symptoms

Pale, smooth, or shiny vaginal epithelium

Loss of skin elasticity or turgor

Sparse pubic hair

Dry labia

Friable, unrugated epithelium

Pelvic organ prolapse

Rectocele

Vulvar dermatoses

Vulvar lesions

Vulvar patch erythema

Petechiae of epithelium
Introital stenosis

condition is a decrease in the percentage of superficial cells, with an increase in the percentage of intermediate and parabasal cells. This decrease in glycogen-rich cells inhibits lactobacilli and lactic acid production and increases vaginal $\mathrm{pH}$, changing the natural microflora and rendering the vagina more susceptible to bacterial and fungal infections. ${ }^{1,13}$

In addition to hypoestrogenism, other factors may also cause AV. Cigarette smoking has a direct effect on the vaginal epithelium, reducing estrogen bioavailability, diminishing blood perfusion, and increasing atrophic changes. ${ }^{13}$ Another factor in AV is the level of different androgens, and it has been suggested that postmenopausal women with higher levels of androgens who maintain sexual activity have fewer atrophic changes. ${ }^{15}$ Finally, more severe symptoms of vaginal atrophy have been shown to manifest in women who have never given birth vaginally compared with those who have. ${ }^{1,4,16}$

\section{Presentation}

The normal vaginal environment depends on a delicate physiological balance of hormonal and bacterial interaction. Factors necessary to achieve this equilibrium are normal estrogen secretion, which maintains the glycogen-rich squamous epithelium, and the presence of lactobacilli, which metabolize glycogen to form lactic acid that in turn maintains a normal vaginal $\mathrm{pH}$ between 3.5 and $4.5 .{ }^{1,9}$

Clinical urogenital atrophy manifests as vaginal symptoms that predispose to sexual dysfunction and lower urinary tract symptoms. The vaginal symptoms include dryness, itching, vulvar pruritus, feeling of pressure, leukorrhea, dyspareunia, and yellow malodorous discharge (Table 1). ${ }^{1,9,13,16}$ Urinary urgency, polyuria, bladder instability, and incontinence may accompany the vaginal complaints. All symptoms of AV can be exacerbated by a simultaneous infection of candidiasis, trichomoniasis, or bacterial vaginosis. The first symptoms may occur before signs noted in the pelvic examination; one of them often is sexual dysfunction and the associated emotional distress. ${ }^{1,4,9,13}$

\section{Diagnosis}

It is important not to assume a diagnosis of AV in every postmenopausal patient who experiences urogenital complaints. A complete medical history should be obtained, followed by a pelvic examination. The appearance of a pale, smooth, and shiny epithelium is a sign of atrophy. Often, inflammation with patchy erythema, petechiae, and increased friability may be present. Signs of AV on the external genitalia include diminished elasticity, skin turgor, sparse pubic hair, dry labia, vulvar dermatoses, vulvar lesions, and fusion of labia minora. ${ }^{1}$

Table 2 lists the laboratory tests useful in the diagnosis of $\mathrm{AV}$, particularly in those cases where diagnosis based on examination is uncertain. These should include serum hormone level assessment and a Papanicolaou test. Histological and cytological findings show a decreased percentage of superficial cells and increased proportion of parabasal cells in the upper one third of the vagina. ${ }^{1}$ An increased $\mathrm{pH}$ exceeding 5.0, as monitored by a $\mathrm{pH}$ strip, may also be a sign of AV. Microscopic examination may reveal evidence of bacterial or fungal infection. The vaginal maturation index obtained through vaginal cytological examination also provides information about the estrogen status. The maturation index is calculated from the total numbers of parabasal, intermediate, and superficial epithelial cells per 100 cells on the vaginal smear; an increase in parabasal and intermediate cells is observed with hypoestrogenemia compared with premenopause levels. ${ }^{13}$ However, although the maturation index has proven to be a useful measure in clinical studies, it is not routinely used in clinical practice. An examination of the cervix and the pelvis for masses also should be performed. $1,4,9,13,17$

\section{Treatment}

Current recommendations from the American College of Obstetricians and Gynecologists (ACOG) indicate that women with moderate to severe AV may be offered vaginal estrogen after a thorough discussion of its risks and benefits. ${ }^{18}$ Similar recommendations have been proposed by The Society of Obstetricians and Gynecologists of Canada (SOGC), which also recommends the use of lubricants or moisturizers and maintenance of sexual activity. ${ }^{2}$

Table 2. Laboratory Diagnosis of Atrophic Vaginitis ${ }^{1,9,17}$

Laboratory test

Wet preparation/cytological smear of cells from upper one third of vagina

Serum hormone concentration

Vaginal $\mathrm{pH}$

Microscopy
Positive indication

Atrophic cytological changes, including increase in proportion of parabasal cells

Low level of circulating estrogen $(\leq 4.5)$

$\mathrm{pH}$ elevation above normal postmenopausal levels $(\mathrm{pH}>5)$

Elimination of diagnosis of trichomoniasis, candidiasis, and bacterial vaginosis 


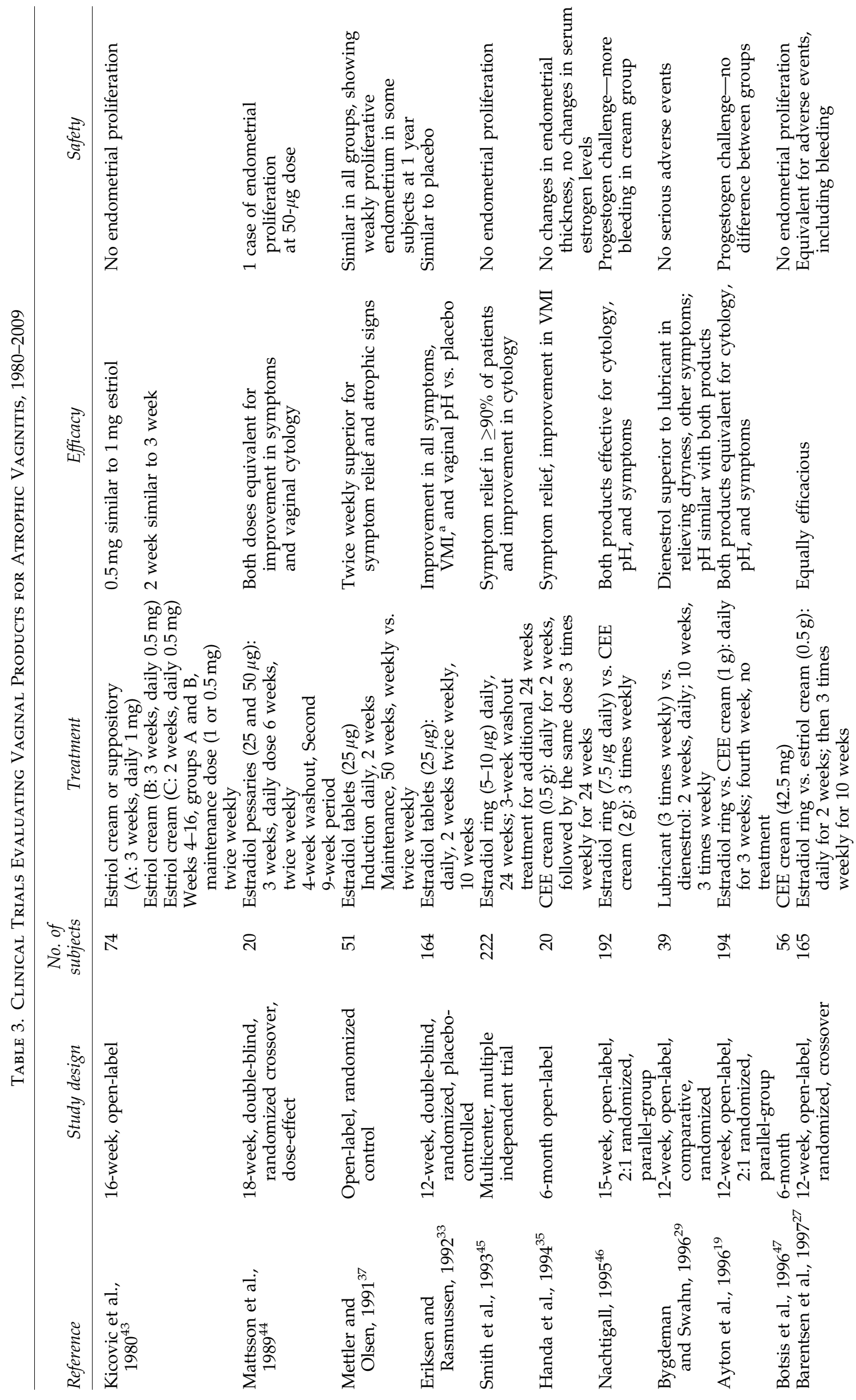




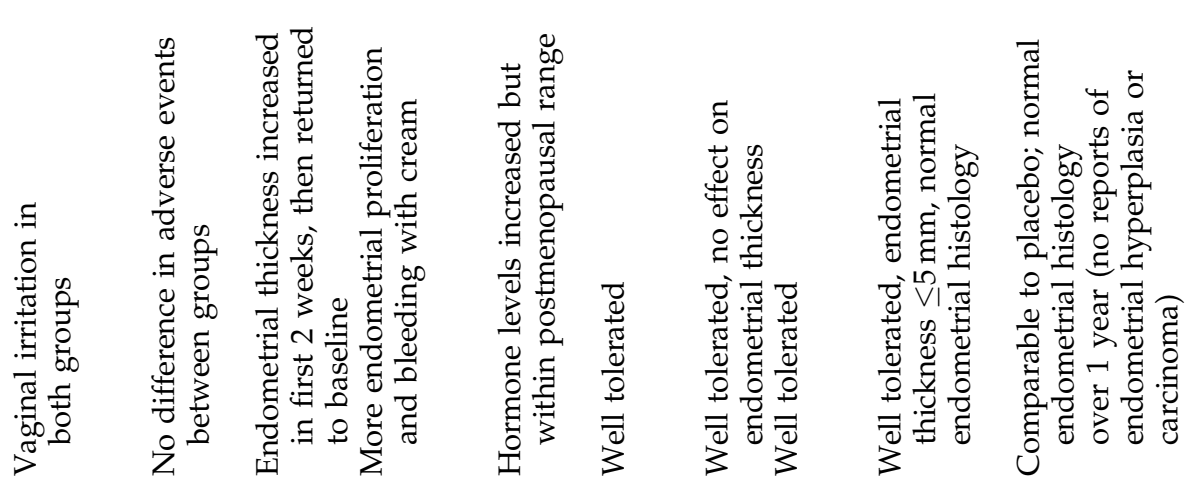

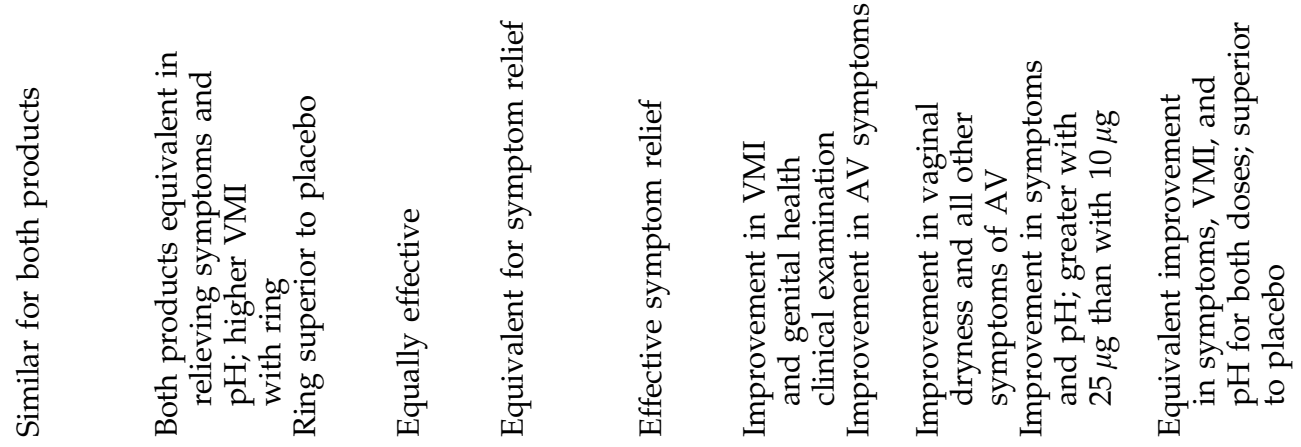

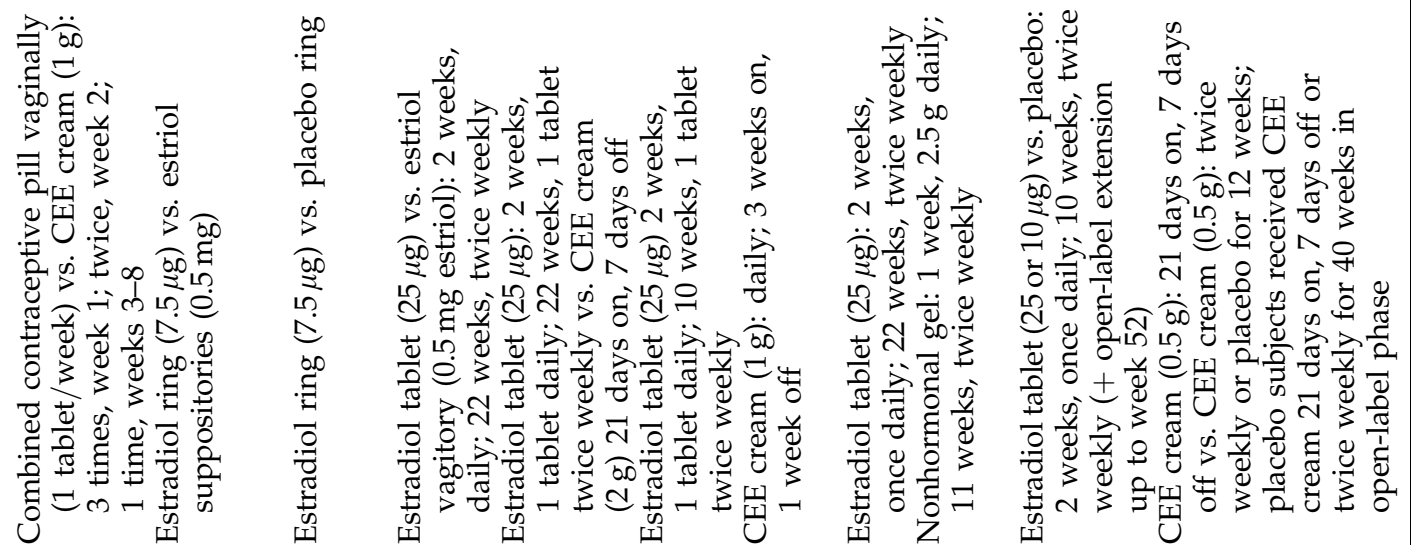

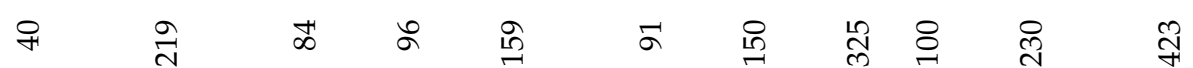

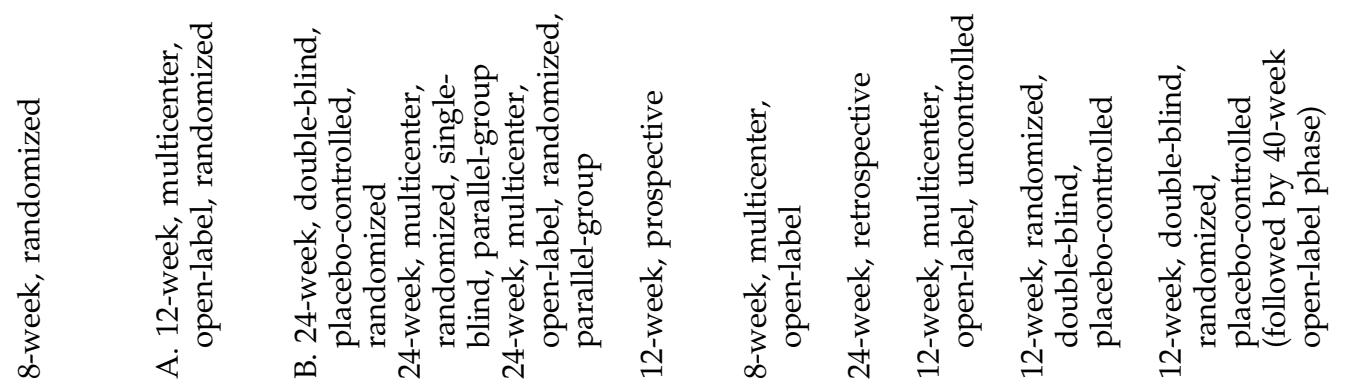

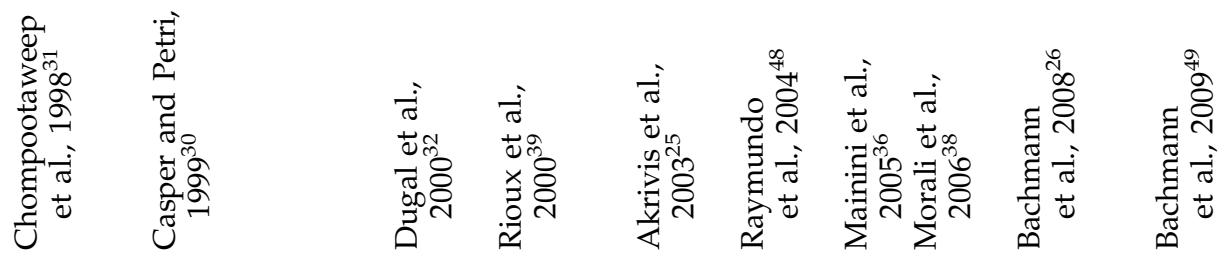




\section{Estrogen therapy}

Estrogen therapy has proven to be effective in the restoration of vaginal anatomy along with resolution of symptoms. Although both orally and vaginally administered estrogen therapies have been shown to be effective for the relief of vaginal dryness, ${ }^{19,20}$ a meta-analysis of 10 clinical trials showed that vaginal estrogen therapy provided the greatest symptom relief and improvement of vaginal/vulvar atrophy. ${ }^{8}$ The advantages of systemic administration include prevention of postmenopausal osteoporosis and alleviation of vasomotor symptoms along with relief of symptoms of AV; however, standard doses of systemic estrogen may not be sufficient to eliminate $\mathrm{AV}$, and simultaneous administration of a local vaginal product may be necessary. ${ }^{14,21}$

In the absence of severe climacteric symptoms, localized vaginal therapy alone, rather than systemic therapy, has been shown to be more appropriate. ${ }^{2,22}$ Estrogen therapy restores normal $\mathrm{pH}$ levels and thickens and revascularizes the epithelium. The number of superficial cells is increased, and existing symptoms are alleviated. Initiating therapy at the time of menopause may prevent the development of urogenital symptoms. Several local vaginal products, such as creams, tablets, suppositories, and rings, are available for the treatment of AV. Creams and tablets are inserted using an applicator, and rings are placed manually. An important aspect of treatment with low-dose vaginal estrogen is its efficacy in relieving symptoms without causing significant proliferation of the vaginal epithelium. ${ }^{14,21,23}$ Topical estrogen treatment has also been shown to lower genitourinary $\mathrm{pH}$, decreasing the incidence of UTIs. Improved epithelial vascularity associated with hormonal treatment leads to increasing systemic absorption of estrogen with ongoing treatment.

Some of the negative effects of vaginal treatment include patient dislike of vaginal manipulation and irregular treatment intervals with creams, tablets, and suppositories. Topical estrogens may be applied cyclically or symptomatically, making it more difficult for patients to remember to apply treatments. ${ }^{1}$ Vaginal rings can be used for a period of 3 months, during which a constant supply of the hormone is maintained. ${ }^{1}$

\section{Moisturizers and lubricants}

Products used as lubricants include Replens ${ }^{\circledR}$ (WarnerLambert, Morris Plains, NJ) and K-Y ${ }^{\circledR}$ Jelly (Johnson \& Johnson, Skillman, NJ). These may be used in combination with estrogens or as alternatives. Some patients may choose not to take estrogen therapy, have medical contraindications, or experience adverse events. Moisturizers provide lubrication, help coital comfort, and can normalize vaginal $\mathrm{pH}$; however, they do not treat the underlying cause of the condition but merely provide temporary relief of symptoms. The length of effectiveness is generally less than 24 hours. ${ }^{1,24}$

\section{Sexual activity}

There is a positive link between sexual activity and maintenance of vaginal elasticity and pliability, in addition to lubricative response to sexual stimulation. ${ }^{1}$ Women who participate in sexual activity report fewer symptoms of AV and, on vaginal examination, have less evidence of atrophy compared with sexually inactive women. ${ }^{15}$ Interestingly, women with less AV on examination have also been shown to have significantly higher mean circulating levels of androgens and gonadotropins compared with those with greater symptoms of $\mathrm{AV} .^{15}$

\section{Vaginal Estrogen Products}

Several clinical trials have examined the use of estrogen preparations for the treatment of AV (Table 3), ${ }^{19,24-40}$ and a few reviews have also been published. ${ }^{23,41,42}$ The studies have been randomized controlled trials that measured efficacy, safety, or both. Although the overall quality of trials has been good, not all trials measured the same outcomes. Comparison of the efficacy of estrogen preparations in relieving symptoms of AV showed findings favoring creams, rings, and tablets. Several trials assessed safety and evaluated the occurrence of endometrial hyperplasia, endometrial stimulation, and other adverse events, such as breast tenderness and uterine bleeding. Acceptability of dosage forms has also been studied to compare the comfort and ease of product use, overall product rating, delivery system, and satisfaction.

Many studies that examined vaginal products were not blinded because of obvious differences in product characteristics (Table 3). ${ }^{23}$ Each preparation was used in multiple studies; however, the doses, schedules, and outcomes for each preparation were not the same across all studies. Individual dosage forms are discussed here to highlight the advantages and disadvantages of each. Clinical data for each form are discussed with respect to efficacy, safety, and acceptability of the treatment. ${ }^{23}$

Efficacy in these studies was measured by assessment of vaginal $\mathrm{pH}$, health, and dryness and improvement in cytology, maturation value, karyopyknotic index, and vaginal atrophy. Both participant and physician assessments were used to measure relief of symptoms associated with vaginal atrophy. ${ }^{42}$ Safety was reported as incidence of hyperplasia, measurement of endometrial proliferation, endometrial thickness of $>5 \mathrm{~mm}$, incidence of bleeding after progestogen challenge test, blood estradiol levels, and withdrawal due to adverse events. Acceptability was measured by adherence to treatment and product preference. ${ }^{42}$

\section{Vaginal rings}

Two commonly used products are Estring ${ }^{\circledR}$ (Pharmacia \& Upjohn, New York, NY) and Femring ${ }^{\circledR}$ (Warner Chilcott, Larne, U.K.). The major advantage of vaginal rings is the minimized frequency of application because of the extended release of the hormone over a period of 3 months. Vaginal rings can be worn during coitus and, if dislocated, can be adjusted back into position. Vaginal rings have been reported to fall out occasionally, however, in which case it is recommended that the ring be washed with a mild soap and lukewarm water and be reinserted. A burst release of the hormone is often obtained before the release is stabilized, with maximal estradiol plasma concentrations obtained within the first 3 hours after insertion. ${ }^{23}$

Estradiol vaginal ring (Estring). The estradiol vaginal ring is a slightly opaque ring, $55 \mathrm{~mm}$ in diameter, with a core containing a drug reservoir of $2 \mathrm{mg} 17 \beta$-estradiol surrounded by an outer silicone sheath. ${ }^{50}$ It is indicated for the treatment of urogenital symptoms associated with postmenopausal 
atrophy of the vagina (such as dryness, burning, pruritus, and dyspareunia) or the lower urinary tract (such as urinary urgency and dysuria). When placed in the vagina, the ring releases approximately $7.5 \mu \mathrm{g}$ of $17 \beta$-estradiol in 24 hours in a consistent, stable manner for 90 days. All the vaginal ring studies cited in Table 3 used either Estring or a ring providing a similar dose of $17 \beta$-estradiol. Estradiol rings, which have been compared with conjugated equine estrogens (CEE) cream, estriol cream, and estriol pessaries, have been shown to successfully alleviate many symptoms of $\mathrm{AV} .^{50}$ Relief of symptoms has been shown to be similar to that obtained with CEE cream. ${ }^{23}$ Other vaginal symptoms, including dryness and dyspareunia, were shown to be relieved to the same extent with estradiol rings as with CEE cream, estriol cream, or estriol pessary. ${ }^{23}$ Estradiol rings relieved pruritus better than estriol creams and as well as pessaries. Objective signs, such as vaginal mucosal maturation, atrophic signs, and vaginal $\mathrm{pH}$, were equally improved with the ring and creams, but the ring was more effective than pessaries. Rings were superior to placebo or no treatment in improving signs and symptoms of $\mathrm{AV}^{23}$

In two pivotal controlled clinical studies comparing the estradiol ring with CEE cream, no difference in efficacy between treatment groups was found with respect to improvement in the assessment of vaginal symptoms after 12 weeks of treatment. ${ }^{19,46}$ In these studies, both products were comparable in reducing vaginal $\mathrm{pH}$ levels and maturing the vaginal mucosa. In one of the studies, moderate endometrial proliferation was noted in 5\% of patients treated with the ring, compared with mild proliferation noted in $10 \%$ of patients treated with CEE cream. ${ }^{46}$

Estradiol acetate vaginal ring (Femring). The estradiol acetate vaginal ring is an off-white, soft, flexible ring with a central core containing estradiol acetate and is available in two strengths. ${ }^{51}$ The $0.05 \mathrm{mg} /$ day estradiol acetate ring has a central core that contains $12.4 \mathrm{mg}$ of estradiol acetate, which releases at a rate equivalent to $0.05 \mathrm{mg} /$ day of estradiol for 3 months, and the $0.1 \mathrm{mg} /$ day ring has a central core that contains $24.8 \mathrm{mg}$ of estradiol acetate, which releases at a rate equivalent to $0.1 \mathrm{mg} /$ day of estradiol for 3 months. Drug release from the estradiol acetate ring is rapid for the first hour and then declines to a relatively constant rate for the rest of the 3-month period. Estradiol acetate and estradiol are rapidly absorbed through the vaginal mucosa as evidenced by time to maximum plasma concentration $\left(t_{\max }\right)$ values for estradiol of $<1$ hour. Following maximum plasma concentration $\left(\mathrm{C}_{\max }\right)$, serum estradiol concentrations decrease rapidly so that by $24-48$ hours postdose, the concentrations are relatively constant through the end of the 3-month dosing interval. ${ }^{51}$

The efficacy of the vaginal ring in relieving vasomotor symptoms and vulvar and vaginal atrophy was studied in a 13-week double-blind, placebo-controlled clinical trial of 333 postmenopausal women. ${ }^{51}$ Patients were randomized to receive either placebo, $0.05 \mathrm{mg} /$ day estradiol acetate ring, or $0.1 \mathrm{mg} /$ day estradiol acetate ring, and statistically significant improvement in the relief of symptoms was seen in both active treatment groups relative to placebo. An increase in vaginal superficial cells with a corresponding reduction in parabasal cells was observed in both treatment groups. A decrease in vaginal $\mathrm{pH}$ was also seen in the two treatment groups. ${ }^{51}$ The estradiol acetate ring is indicated for the treatment of both moderate to severe vasomotor symptoms and vulvar and vaginal atrophy associated with menopause.

\section{Vaginal tablets}

In this section, all solid dosage forms, such as tablets, vagitories, and suppositories, are included. Although these products provide ease of use, retention in the vagina may be difficult for patients with prolapse. Further, there may be an initial need to use a nonhormonal lubricant or moisturizer for the immediate relief of vaginal dryness.

Estradiol tablets $\left(\right.$ Vagifem $\left.^{\circledR}\right)$. Estradiol tablets (Vagifem, Novo Nordisk, Princeton, NJ) are small, white film-coated tablets each containing an equivalent of $25 \mu \mathrm{g}$ estradiol for the treatment of $A V .{ }^{52}$ Each tablet is inserted into the vagina using an applicator provided with the product. The initial dose is one estradiol tablet inserted vaginally once daily for 2 weeks, followed by a maintenance dose of one tablet inserted vaginally twice a week.

Clinical trials have evaluated the efficacy and safety of estradiol tablets compared with placebo and CEE cream. In a placebo-controlled, randomized, 12-week trial, patients inserted one tablet vaginally daily for 14 days, followed by one tablet twice weekly for the remaining 10 weeks. ${ }^{33}$ Vaginal estradiol tablets were found to be effective in relieving vaginal dryness, soreness, and irritation associated with AV. The improvement in symptoms was seen at week 2 and maintained through week 12 . Adverse events were generally mild and included vulval itching, vaginal discharge, and skin rash. $^{33}$ In a prospective, open-label, randomized, parallelgroup, 24-week study that evaluated vaginal estradiol tablets compared with CEE cream, the efficacy of tablets in relieving AV was found to be similar to that of CEE cream. ${ }^{39}$ Endometrial hyperplasia was reported in 2 patients using the vaginal cream and no patients using vaginal tablets. Adverse events were generally more frequent with the vaginal cream, and patients found the estradiol tablets to be more acceptable and easier to use than the cream.

Among 96 women treated with either $17 \beta$-estradiol vaginal tablets $(25 \mu \mathrm{g})$ or estriol vagitories $(0.5 \mathrm{mg})$ for 24 weeks (daily for 2 weeks and twice weekly thereafter) in a randomized, parallel-group, single-blind, multicenter trial, both treatments were found to be equally effective in treating the symptoms of $\mathrm{AV}^{32}$; however, the tablets produced less leakage, with less need for sanitary protection. This dosage form was found to be more acceptable by patients and was expected to improve compliance. Endometrial thickness increased during the first 2 weeks in both groups but returned to baseline by the end of the trial. ${ }^{32}$

In an open-label, randomized controlled study, 51 postmenopausal women ( $\leq 70$ years of age) were treated with $17 \beta$ estradiol vaginal tablets $(25 \mu \mathrm{g})$ daily for 2 weeks, then received either once-weekly or twice-weekly treatment for an additional 50 weeks. ${ }^{37}$ At the end of the study, improvement of all signs and symptoms of AV was seen in both groups, although significantly better results were seen in the group receiving twice-weekly vs. once-weekly treatment. Only 1 patient in the once-weekly group and 2 patients in the twiceweekly group showed a weakly proliferative endometrium at the end of the study. ${ }^{37}$ 
A 12-week, multicenter, randomized, double-blind, parallel-group study evaluated the efficacy of vaginal tablets containing estradiol 10 or $25 \mu$ g compared with placebo. ${ }^{26}$ Both doses of estradiol were more effective than placebo at relieving vaginal symptoms (dryness, soreness, and irritation), improving urogenital atrophy, decreasing vaginal $\mathrm{pH}$, and increasing maturation of the vaginal and urethral epithelium. Improvements were greater with the $25-\mu \mathrm{g}$ estradiol dose compared with the 10- $\mu \mathrm{g}$ estradiol dose. Thus, the $25-\mu \mathrm{g}$ dose of estradiol was used during the 40-week open-label study extension period, during which efficacy was maintained.

\section{Vaginal creams}

Estrogen-containing vaginal creams include Premarin ${ }^{\circledR}$ Vaginal Cream (Wyeth, Philadelphia, PA) and Estrace ${ }^{\circledR}$ (Warner Chilcott, Rockaway, NJ). Creams are currently the most common choice of vaginal product for the treatment of $\mathrm{AV}$ and provide flexibility of dosage and frequency of administration.

Conjugated estrogens cream (Premarin Vaginal Cream). CEE cream is a nonliquefying cream indicated for the localized treatment of AV and kraurosis vulvae. ${ }^{53}$ Each gram of CEE cream contains $0.625 \mathrm{mg}$ of conjugated estrogens, which is a mixture of estrone, equilin, $17 \beta$-dihydroequilin, $17 \alpha$-estradiol, and $17 \alpha$-dihydroequilin. CEE cream should be used cyclically ( 3 weeks on, 1 week off) at the lowest dose necessary to control symptoms $\left(0.5-2.0\right.$ g cream daily), ${ }^{53}$ although in clinical practice, it is often used on a maintenance schedule of one to three doses per week. ${ }^{2,54} \mathrm{CEE}$ cream is the most extensively investigated vaginal product and has a longterm safety record. ${ }^{19,35,39,42,46,49}$ Comparative trials have demonstrated that CEE cream is as effective as vaginal rings and tablets in the treatment of AV (Table 3). ${ }^{19,39,46}$ In addition, CEE cream has been shown to be as effective as vaginally applied oral contraceptive tablets in reducing the symptoms of vaginal dryness, dyspareunia, and irritation. ${ }^{31}$ CEE cream has also been shown to be effective in reducing vaginal $\mathrm{pH}$ and improving mucosal maturation (Table 3). ${ }^{19,27,35}$

Systemic hormone levels have been investigated with the use of CEE cream. In one study comparing vaginal estradiol tablets $25 \mu \mathrm{g}$ with CEE cream $2 \mathrm{~g}$ (equivalent to CEE $1.25 \mathrm{mg}$ ) daily for a period of 6 months, more patients on CEE cream experienced serum estradiol levels above the normal postmenopausal range vs. those on tablets. ${ }^{39}$ However, it is important to note that this study used a lower maintenance dose of estradiol in tablet form compared with a consistently high dose of CEE daily. In another 6-month study of 20 women with objective signs and symptoms of $\mathrm{AV}$ receiving CEE cream $0.5 \mathrm{~g}$ (equivalent to CEE $0.3 \mathrm{mg}$ ) three times a week, serum levels of estradiol were detectable in only 5 of the 20 subjects, 2 of whom had detectable levels before treatment as well. $^{35}$

Although such adverse events as endometrial proliferation, uterine bleeding, perineal pain, and breast pain and tenderness have been reported more often with the use of CEE cream compared with rings or tablets, these have been relatively mild. ${ }^{42}$ In addition, higher rates of adverse events may be related to the higher hormonal dose of CEE used in the studies. CEE cream was often used at a higher dose per day ( $1 \mathrm{~g}$ [equivalent to CEE $0.625 \mathrm{mg}$ ] or more) compared with estradiol $(5-25 \mu \mathrm{g})$ in rings or tablets. ${ }^{42}$ When lower doses of CEE cream were examined ( $0.5 \mathrm{~g}$ [equivalent to CEE $0.3 \mathrm{mg}$ ] three times a week), symptomatic relief was obtained with no significant changes in endometrial thickness or uterine artery resistance. ${ }^{35}$ Low-dose CEE cream has been shown to provide local vaginal effects with limited or no measurable changes in systemic markers. ${ }^{55}$

Most recently, a randomized, double-blind, placebocontrolled study $(n=423)$ evaluated the efficacy and safety of low-dose CEE cream ( $0.5 \mathrm{~g}$ [equivalent to CEE $0.3 \mathrm{mg}$ ]) administered daily or twice weekly (an intermittent regimen often prescribed in clinical practice) in treating postmenopausal women with moderate to severe AV. ${ }^{49}$ After 12 weeks of therapy, both regimens showed significantly greater improvements in vaginal maturation index, vaginal $\mathrm{pH}$, and most bothersome symptom score compared with placebo. ${ }^{49}$ These improvements were sustained over the open-label phase (remaining 40 weeks), in which women in the placebo groups received low-dose $\mathrm{CEE}$ cream at their respective active treatment regimens. Both low-dose regimens were found to be safe and well tolerated, with minimal differences in the frequency and type of adverse events between the active and placebo groups and between regimens during the open-label phase. ${ }^{49}$ Taken together, lower and less frequent dosing with CEE cream provides safe and effective relief of AV in postmenopausal women.

Estradiol vaginal cream (Estrace). Estradiol vaginal cream is a nonliquefying cream containing $0.01 \%$ estradiol and is indicated for the localized treatment of vulval and vaginal atrophy. ${ }^{56}$ In a single-blind, single-arm clinical study, various doses of estradiol cream $(1.25,2.5,5,10 \mu \mathrm{g})$ were investigated for the relief of symptoms of urogenital atrophy in postmenopausal women. ${ }^{40}$ At the $10-\mu \mathrm{g}$ dose, all patients responded to treatment. Vaginal cytology showed improvement at 3 and 12 weeks, vaginal $\mathrm{pH}$ decreased from postmenopausal to premenopausal levels, and the endometrium remained atrophic. Circulating estradiol levels were within postmenopausal range. ${ }^{40}$

Estriol cream. Estriol cream has been shown to be as effective as an estradiol ring in decreasing vaginal $\mathrm{pH}$, dryness, and other signs of atrophy, as well as improving vaginal maturation. ${ }^{27}$ However, estriol cream did not relieve pruritus as well as the estradiol ring. The incidences of vaginal bleeding and other drug-related adverse events have been reported to be similar with estriol cream and the estradiol ring. ${ }^{27}$

In a study comparing vaginal estriol cream (1 or $0.5 \mathrm{mg} /$ day for 3 weeks) with vaginal estriol suppositories $(0.5 \mathrm{mg} /$ day for 2 weeks) followed by a twice-weekly maintenance dose, a sharp rise in the plasma estriol level was obtained, followed by a gradual decline. ${ }^{43}$ Estradiol and estrone levels remained unchanged, and no endometrial proliferation was reported. ${ }^{43}$ In another study of 11 postmenopausal women treated with vaginal estriol cream $(0.5 \mathrm{mg} /$ day for 3 weeks, then twice weekly for 5 weeks), a slight suppression of follicle-stimulating hormone (FSH) and luteinizing hor- 
mone (LH) levels was noted, although there was no change in circulating levels of estradiol or estrone. ${ }^{57}$

\section{Systemic absorption of vaginal estrogens}

Applied estrogens are water soluble and readily absorbed through the mucous membranes. Once absorbed, exogenous estrogens are distributed and metabolized in the body in the same manner as endogenous estrogens. ${ }^{56}$ The primary concern for postmenopausal women is the risk of endometrial hyperplasia resulting from systemic effects of estrogens. Systemic absorption may also lead to such adverse effects as headache, breast tenderness, and vaginal bleeding. ${ }^{58}$

In a 12-week study comparing the vaginal estradiol ring with CEE cream, 8 of $131(6 \%)$ women using the ring and 5 of $63(8 \%)$ women using the cream reported mild vaginal bleeding. ${ }^{19}$ A proliferative endometrium was reported by 1 woman in each treatment group among those who experienced bleeding, and there were no reports of endometrial hyperplasia. Serum hormone levels were not assessed in this study.

In a study of long-term use of estradiol tablets in 51 women, there were 3 patients with weak endometrial proliferation after 1 year. ${ }^{37}$ Among the 9 women who continued treatment for 2 years, there were no reports of endometrial proliferation. Small changes observed in serum levels of estradiol, $\mathrm{FSH}$, and LH were all within the postmenopausal range. ${ }^{37}$

There have been no significant differences reported between treatment methods for endometrial hyperplasia or proliferation. ${ }^{42}$ In one study comparing CEE cream $2 \mathrm{~g}$ (equivalent to CEE $1.25 \mathrm{mg}$ ) with vaginal estradiol tablets $25 \mu \mathrm{g}$, endometrial proliferation and hyperplasia were reported in more patients using the CEE cream (13 patients) compared with tablets (1 patient). ${ }^{39}$ In a study of a lower dose of CEE cream $0.5 \mathrm{~g}$ (equivalent to CEE $0.3 \mathrm{mg}$ ), however, there were no significant changes in endometrial thickness or serum estrogen levels after 6 months of use. ${ }^{35}$ In the recent 1-year randomized controlled study evaluating two commonly prescribed regimens of low-dose CEE cream $0.5 \mathrm{~g}$ (equivalent to CEE $0.3 \mathrm{mg}$ ) in postmenopausal women $(n=423)$ with moderate to severe AV, endometrial histology results indicated a proliferative endometrium for 6 subjects each with the daily and twice-weekly regimens. ${ }^{49}$ There were no cases of endometrial hyperplasia or carcinoma reported during the 52-week study period. Overall, the incidence of vaginal bleeding was very low during the study, 6 $(1.4 \%)$ and $4(1.0 \%)$ subjects during the double-blind and open-label phases, respectively.

In a study of estradiol cream (10 $\mu$ g daily for 3 weeks, followed by twice-weekly dosing for 9 weeks), there were no reports of endometrial hyperplasia, and serum estrogen levels remained within the postmenopausal range during 12 weeks of use. ${ }^{40}$ In studies of estriol vaginal cream $(1$ or $0.5 \mathrm{mg}$ per day), increases in plasma estriol levels were observed during the first day of treatment, followed by a gradual decline. 43,57 Estriol does not appear to promote endometrial proliferation when administered orally, and there were no reported cases of endometrial hyperplasia or changes in serum estrogen in these studies of vaginal administration. Comparative studies have reported significant changes in blood estradiol levels, although still within normal postmenopausal range, with the ring showing lower levels than the tablet (Weissberg cited in reference 41) and the tablet showing lower levels than CEE cream. ${ }^{39}$ This last difference has been ascribed by some authors to different dosing schedules between the tablet and CEE cream. ${ }^{23}$

\section{Breast cancer risk}

It has been shown that the risk of breast cancer increases with oral use of combined estrogen-progestin therapy beyond 3-5 years, although the absolute risk is considered rare. ${ }^{59}$ The Women's Health Initiative (WHI) study showed a small but significant increase in the risk of invasive breast cancer with combined estrogen-progestin therapy (follow-up of 5.6 years) ${ }^{60}$ Results of the WHI study ${ }^{61}$ and Women's Health Study ${ }^{62}$ showed no increase in breast cancer risk in women receiving oral estrogen monotherapy. There is currently a lack of evidence on the risk of breast cancer associated with vaginal estrogen therapy. ${ }^{54}$ Similar to transdermal therapy, localized administration of vaginal estrogens bypasses firstpass metabolism by the liver, ${ }^{58,63,64}$ limiting systemic exposure. $^{23,65}$ Results of the Million Women Study showed that although the risk of breast cancer in women treated with transdermal hormone therapy was significantly greater compared with never users, the risk was moderately lower than that in women using oral hormone therapy (relative risk 1.24 vs. 1.32 , respectively). ${ }^{52}$ The use of oral and transdermal estradiol was associated with a comparable risk of breast cancer in another cohort study. ${ }^{66}$ However, a recent European prospective cohort study found that users of oral hormone therapy had a significantly higher risk of breast cancer compared with women treated with transdermal therapy (both estrogen and estradiol: hazard ratio 2.14 vs. 1.27 , respectively, $p<0.01){ }^{67}$ Further studies are needed to clarify the risk of breast cancer associated with vaginal estrogens, as well as the risk of recurrence in breast cancer survivors. ${ }^{54,68}$

\section{Current Status of Estrogen Therapy in Postmenopausal Women}

Although the use of vaginal estrogen products is recommended for the treatment of AV and other urogenital conditions, it should be noted that because of the general risks associated with estrogen use, estrogens with or without progestins should be prescribed at the lowest effective doses for the shortest duration consistent with the treatment goals and risks for the individual woman. ${ }^{22}$ It should be noted that tailored treatment programs for women with AV may require low-dose estrogens for extended periods of time. Close clinical surveillance of all women taking estrogens is particularly important. Systemic administration of unopposed estrogens has been reported to increase the risk of endometrial carcinoma in postmenopausal women who have an intact uterus. ${ }^{69}$ Local administration of vaginal estrogen formulations remains a preferred option for the sole treatment of vaginal symptoms. ${ }^{59}$

Current recommendations from the $\mathrm{ACOG}^{18}$ and $\mathrm{SOGC}^{2}$ indicate that women with severe AV may be offered vaginal estrogen after a thorough discussion of its risks and benefits. Further, very low doses of estrogen, such as $0.3 \mathrm{mg}$ of CEE administered vaginally as seldom as twice a week, may be used to relieve symptoms of moderate to severe $\mathrm{AV} .{ }^{49}$ The vaginal doses may be associated with a very low incidence of 
endometrial proliferation, ${ }^{23,35}$ but a recent study of low-dose CEE cream demonstrated endometrial safety over 1 year of use. ${ }^{49}$ Although studies with estrogen tablets and vaginal rings suggest lower systemic hormone absorption, these studies do not provide sufficient data to recommend them as alternatives for AV treatment.

\section{Conclusions}

Almost all hormone treatment regimens incorporating estrogen, with or without progestin, have produced evidence of subjective improvement of $\mathrm{AV}$, including reduced vaginal dryness, irritation, pruritus, and dyspareunia. Many studies have also demonstrated improvement in objective parameters of $\mathrm{AV}$, including improvement in vaginal maturation and karyopyknotic indices.

Vaginal atrophy need not be an inevitable consequence of menopause or other events that cause long-term estrogen loss for women. Active diagnosis and intervention may prevent development of AV or eliminate existing symptoms. Menopausal estrogen therapy is a current option, and awareness of the many choices for delivery of estrogen greatly increases physicians' ability to prescribe a treatment that is better tailored to patients' needs and expectations.

\section{Acknowledgments}

Editorial support for the writing of this article was provided by Bo Choi, Ph.D., and funded by Wyeth Pharmaceuticals, Collegeville, PA. The author was not compensated and retained full editorial control over the content.

\section{Disclosure Statement}

C.L. has served on the Speaker's Bureau for Wyeth, Novartis, GlaxoSmithKline, Pfizer, and Berlex.

\section{References}

1. Bachmann GA, Nevadunsky NS. Diagnosis and treatment of atrophic vaginitis. Am Fam Physician 2000;61:30903096.

2. Society of Obstetricians and Gynaecologists of Canada. SOGC clinical practice guidelines. The detection and management of vaginal atrophy. Number 145, May 2004. Int J Gynaecol Obstet 2005;88:222-228.

3. Kelley C. Estrogen and its effect on vaginal atrophy in postmenopausal women. Urol Nurs 2007;27:40-45.

4. Carcio H. Urogenital atrophy. A new approach to vaginitis diagnosis. Adv Nurse Pract 2002;10:40-48, 51.

5. Nilsson K, Risberg B, Heimer G. The vaginal epithelium in the postmenopause-Cytology, histology and $\mathrm{pH}$ as methods of assessment. Maturitas 1995;21:51-56.

6. Bachmann G, Ebert GA, Burd ID. Vulvovaginal complaints. In: Lobo RA, ed. Treatment of the postmenopausal woman: Basic and clinical aspects. Philadelphia: Lippincott Williams \& Wilkins, 1999:195-201.

7. Willhite LA, O'Connell MB. Urogenital atrophy: Prevention and treatment. Pharmacotherapy 2001;21:464-480.

8. Cardozo L, Bachmann G, McClish D, Fonda D, Birgerson L. Meta-analysis of estrogen therapy in the management of urogenital atrophy in postmenopausal women: Second report of the Hormones and Urogenital Therapy Committee. Obstet Gynecol 1998;92:722-727.
9. Pandit L, Ouslander JG. Postmenopausal vaginal atrophy and atrophic vaginitis. Am J Med Sci 1997;314:228-231.

10. Pastore LM, Carter RA, Hulka BS, Wells E. Self-reported urogenital symptoms in postmenopausal women: Women's Health Initiative. Maturitas 2004;49:292-303.

11. Stenberg A, Heimer G, Ulmsten U, Cnattingius S. Prevalence of genitourinary and other climacteric symptoms in 61-yearold women. Maturitas 1996;24:31-36.

12. Dennerstein L, Dudley EC, Hopper JL, Guthrie JR, Burger HG. A prospective population-based study of menopausal symptoms. Obstet Gynecol 2000;96:351-358.

13. Castelo-Branco C, Cancelo MJ, Villero J, Nohales F, Julia MD. Management of postmenopausal vaginal atrophy and atrophic vaginitis. Maturitas 2005;52(Suppl 1):S46-S52.

14. Samsioe G. Urogenital aging-A hidden problem. Am J Obstet Gynecol 1998;178:S245-S249.

15. Leiblum S, Bachmann G, Kemmann E, Colburn D, Swartzman L. Vaginal atrophy in the postmenopausal woman. The importance of sexual activity and hormones. JAMA 1983; 249:2195-2198.

16. Beard MK. Atrophic vaginitis. Can it be prevented as well as treated? Postgrad Med 1992;91:257-260.

17. Osmers R, Volksen M, Schauer A. Vaginosonography for early detection of endometrial carcinoma? Lancet 1990;335: 1569-1571.

18. American College of Obstetricians and Gynecologists Task Force on Hormone Therapy. Genitourinary tract changes. Obstet Gynecol 2004;104:56S-61S.

19. Ayton RA, Darling GM, Murkies AL, et al. A comparative study of safety and efficacy of continuous low dose oestradiol released from a vaginal ring compared with conjugated equine oestrogen vaginal cream in the treatment of postmenopausal urogenital atrophy. Br J Obstet Gynaecol 1996; 103:351-358.

20. Good WR, John VA, Ramirez M, Higgins JE. Comparison of Alora estradiol matrix transdermal delivery system with oral conjugated equine estrogen therapy in relieving menopausal symptoms. Alora Study Group. Climacteric 1999;2:29-36.

21. Rigg LA. Estrogen replacement therapy for atrophic vaginitis. Int J Fertil 1986;31:29-34.

22. North American Menopause Society. Estrogen and progestogen use in peri- and postmenopausal women: March 2007 position statement of the North American Menopause Society. Menopause 2007;14:1-17.

23. Crandall C. Vaginal estrogen preparations: A review of safety and efficacy for vaginal atrophy. J Womens Health 2002;11:857-877.

24. Nachtigall LE. Comparative study: Replens versus local estrogen in menopausal women. Fertil Steril 1994;61:178-180.

25. Akrivis Ch, Varras M, Thodos A, Hadjopoulos G, Bellou A, Antoniou N. Action of $25 \mu \mathrm{g} 17 \beta$-oestradiol vaginal tablets in the treatment of vaginal atrophy in Greek postmenopausal women: Clinical study. Clin Exp Obstet Gynecol 2003;30: 229-234.

26. Bachmann G, Lobo RA, Gut R, Nachtigall L, Notelovitz M. Efficacy of low-dose estradiol vaginal tablets in the treatment of atrophic vaginitis: A randomized controlled trial. Obstet Gynecol 2008;111:67-76.

27. Barentsen $\mathrm{R}$, van de Weijer $\mathrm{PH}$, Schram JH. Continuous low dose estradiol released from a vaginal ring versus estriol vaginal cream for urogenital atrophy. Eur J Obstet Gynecol Reprod Biol 1997;71:73-80.

28. Bottiglione F, Volpe A, Esposito G, Aloysio DD. Transvaginal estriol administration in postmenopausal women: A 
double blind comparative study of two different doses. Maturitas 1995;22:227-232.

29. Bygdeman M, Swahn ML. Replens versus dienoestrol cream in the symptomatic treatment of vaginal atrophy in postmenopausal women. Maturitas 1996;23:259-263.

30. Casper F, Petri E. Local treatment of urogenital atrophy with an estradiol-releasing vaginal ring: A comparative and a placebo-controlled multicenter study. Vaginal Ring Study Group. Int Urogynecol J Pelvic Floor Dysfunct 1999;10:171176.

31. Chompootaweep S, Nunthapisud P, Trivijitsilp P, Sentrakul $\mathrm{P}$, Dusitsin N. The use of two estrogen preparations (a combined contraceptive pill versus conjugated estrogen cream) intravaginally to treat urogenital symptoms in postmenopausal Thai women: A comparative study. Clin Pharmacol Ther 1998;64:204-210.

32. Dugal R, Hesla K, Sordal T, Aase KH, Lilleeidet O, Wickstrom E. Comparison of usefulness of estradiol vaginal tablets and estriol vagitories for treatment of vaginal atrophy. Acta Obstet Gynecol Scand 2000;79:293-297.

33. Eriksen PS, Rasmussen H. Low-dose $17 \beta$-estradiol vaginal tablets in the treatment of atrophic vaginitis: A double-blind placebo controlled study. Eur J Obstet Gynecol Reprod Biol 1992;44:137-144.

34. Gordon WE, Hermann HW, Hunter DC. Treatment of atrophic vaginitis in postmenopausal women with micronized estradiol cream-A follow-up study. J Ky Med Assoc 1979;77:337-339.

35. Handa VL, Bachus KE, Johnston WW, Robboy SJ, Hammond CB. Vaginal administration of low-dose conjugated estrogens: Systemic absorption and effects on the endometrium. Obstet Gynecol 1994;84:215-218.

36. Mainini G, Scaffa C, Rotondi M, Messalli EM, Quirino L, Ragucci A. Local estrogen replacement therapy in postmenopausal atrophic vaginitis: Efficacy and safety of low dose $17 \beta$-estradiol vaginal tablets. Clin Exp Obstet Gynecol 2005;32:111-113.

37. Mettler L, Olsen PG. Long-term treatment of atrophic vaginitis with low-dose oestradiol vaginal tablets. Maturitas 1991;14:23-31.

38. Morali G, Polatti F, Metelitsa EN, Mascarucci P, Magnani P, Marre GB. Open, noncontrolled clinical studies to assess the efficacy and safety of a medical device in form of gel topically and intravaginally used in postmenopausal women with genital atrophy. Arzneimittelforschung 2006;56:230238.

39. Rioux JE, Devlin C, Gelfand MM, Steinberg WM, Hepburn DS. $17 \beta$-estradiol vaginal tablet versus conjugated equine estrogen vaginal cream to relieve menopausal atrophic vaginitis. Menopause 2000;7:156-161.

40. Santen RJ, Pinkerton JV, Conaway M, et al. Treatment of urogenital atrophy with low-dose estradiol: Preliminary results. Menopause 2002;9:179-187.

41. Nothnagle M, Taylor JS. Vaginal estrogen preparations for relief of atrophic vaginitis. Am Fam Physician 2004;69:21112112.

42. Suckling J, Lethaby A, Kennedy R. Local oestrogen for vaginal atrophy in postmenopausal women. Cochrane Database Syst Rev 2006;CD001500.

43. Kicovic PM, Cortes-Prieto J, Milojevic S, Haspels AA, Aljinovic A. The treatment of postmenopausal vaginal atrophy with Ovestin vaginal cream or suppositories: Clinical, endocrinological and safety aspects. Maturitas 1980;2:275282.
44. Mattsson LA, Cullberg G, Eriksson O, Knutsson F. Vaginal administration of low-dose oestradiol-Effects on the endometrium and vaginal cytology. Maturitas 1989;11:217-222.

45. Smith P, Heimer G, Lindskog M, Ulmsten U. Oestradiolreleasing vaginal ring for treatment of postmenopausal urogenital atrophy. Maturitas 1993;16:145-154.

46. Nachtigall LE. Clinical trial of the estradiol vaginal ring in the U.S. Maturitas 1995;22:S43-S47.

47. Botsis D, Kassanos D, Antoniou G, Vitoratos N, Zourlas PA. Transvaginal sonography in postmenopausal women treated with low-dose estrogens locally administered. Maturitas 1996;23:41-45.

48. Raymundo N, Yu-cheng B, Zi-yan H, et al. Treatment of atrophic vaginitis with topical conjugated equine estrogens in postmenopausal Asian women. Climacteric 2004;7:312-318.

49. Bachmann G, Bouchard C, Hoppe D, et al. Efficacy and safety of low-dose regimens of conjugated estrogens cream administered vaginally. Menopause 2009;16:719-727.

50. ESTRING ${ }^{\circledR}$ estradiol vaginal ring $2 \mathrm{mg}$ [package insert]. New York: Pharmacia \& Upjohn Company, Division of Pfizer Inc, 2007.

51. FEMRING ${ }^{\circledR}$ estradiol acetate vaginal ring [package insert]. Rockaway, NJ: Warner Chilcott, Inc., 2008.

52. VAGIFEM ${ }^{\circledR}$ estradiol vaginal tablets [package insert]. Princeton, NJ: Novo Nordisk Pharmaceuticals, Inc, 2003.

53. PREMARIN ${ }^{\circledR}$ conjugated estrogens vaginal cream [package insert]. Philadelphia, PA: Wyeth Pharmaceuticals Inc, 2008.

54. North American Menopause Society. The role of local vaginal estrogen for treatment of vaginal atrophy in postmenopausal women: 2007 position statement of The North American Menopause Society. Menopause 2007;14: 357-369.

55. Mandel FP, Geola FL, Meldrum DR, et al. Biological effects of various doses of vaginally administered conjugated equine estrogens in postmenopausal women. J Clin Endocrinol Metab 1983;57:133-139.

56. ESTRACE ${ }^{\circledR}$ Cream estradiol vaginal cream, USP, $0.01 \%$ [package insert]. Rockaway, NJ: Warner Chilcott, Inc, 2007.

57. Haspels AA, Luisi M, Kicovic PM. Endocrinological and clinical investigations in postmenopausal women following administration of vaginal cream containing oestriol. Maturitas 1981;3:321-327.

58. Balfour JA, Heel RC. Transdermal estradiol. A review of its pharmacodynamic and pharmacokinetic properties, and therapeutic efficacy in the treatment of menopausal complaints. Drugs 1990;40:561-582.

59. North American Menopause Society. Estrogen and progestogen use in postmenopausal women: July 2008 position statement of The North American Menopause Society. Menopause 2008;15:584-602.

60. Anderson GL, Chlebowski RT, Rossouw JE, et al. Prior hormone therapy and breast cancer risk in the Women's Health Initiative randomized trial of estrogen plus progestin. Maturitas 2006;55:103-115.

61. Anderson GL, Limacher M, Assaf AR, et al. Effects of conjugated equine estrogen in postmenopausal women with hysterectomy: The Women's Health Initiative randomized controlled trial. JAMA 2004;291:1701-1712.

62. Zhang SM, Manson JE, Rexrode KM, Cook NR, Buring JE, Lee IM. Use of oral conjugated estrogen alone and risk of breast cancer. Am J Epidemiol 2007;165:524-529.

63. Ansbacher R. The pharmacokinetics and efficacy of different estrogens are not equivalent. Am J Obstet Gynecol 2001;184: 255-263. 
64. Kuhl H. Pharmacokinetics of oestrogens and progestogens. Maturitas 1990;12:171-197.

65. Minkin MJ. Considerations in the choice of oral vs. transdermal hormone therapy: A review. J Reprod Med 2004; 49:311-320.

66. Lyytinen H, Pukkala E, Ylikorkala O. Breast cancer risk in postmenopausal women using estrogen-only therapy. Obstet Gynecol 2006;108:1354-1360.

67. Corrao G, Zambon A, Conti V, et al. Menopause hormone replacement therapy and cancer risk: An Italian record linkage investigation. Ann Oncol 2008;19:150 155.

68. Al-Baghdadi O, Ewies AA. Topical estrogen therapy in the management of postmenopausal vaginal atrophy: An upto-date overview. Climacteric 2009;12:91-105.
69. Creasman WT. Endometrial cancer: Incidence, prognostic factors, diagnosis, and treatment. Semin Oncol 1997;24 (1 Suppl 1):140-150.

Address correspondence to: Catherine Lynch, M.D.

Professor of Obstetrics and Gynecology Director, Division of General Obstetrics and Gynecology Department of Obstetrics and Gynecology

University of South Florida 2 Tampa General Circle Suite 6046 Tampa, FL 33606

E-mail: clynch@hsc.usf.edu 